\title{
REOLOGIA APLICADA A SIMULAÇÃO DO FATOR DE ATRITO PARA O ESCOAMENTO CONFINADO DE CAULIM PELOS MODELOS DE CHURCHILL E DE SWAMEE-JAIN
}

\author{
André Luis Mileo Ferraioli Silva ' \\ Andre Luiz Amarante Mesquita ${ }^{2}$ \\ Augusta Maria Paulain Ferreira Felipe ${ }^{\prime}$ \\ José Antônio da Silva Souza ${ }^{3}$
}

\section{Resumo}

Estimar perdas de carga devido à fricção em tubos fechados é uma tarefa importante na solução de muitos problemas práticos nos diferentes ramos da profissão de engenharia. $O$ projeto hidráulico e análise de sistemas de escoamento de suspensão mineral são dois exemplos. Muitos autores consideram os modelos de Churchill e Swamee-Jain promissor para avaliar as perdas de carga devido ao atrito em condutas de tubos fechados. Para investigar o fator de atrito os modelos de Churchill e Swamee-Jain foram avaliados para os dados reológicos referente ao escoamento confinado de suspensão de caulim para tubulação de ferro galvanizado com 0,I a I,0 metro de diâmetro em faixa de vazão de 0,002 a 0,6 $\mathrm{m}^{3}$ por segundo. Todos os dois modelos apresentaram boa representação do fator de atrito de forma coerente com baixa discrepância relativa percentual proporcionando estimativa de valores para utilização em projeto e analise de sistemas de escoamento de caulim.

Palavras-chave: Suspensão de caulim; Reologia; Fator de atrito.

\section{RHEOLOGY APPLIED TO A FRICTION FACTOR SIMULATION FOR CONFINED DISPOSAL OF KAOLIN BY CHURCHILL MODELS AND SWAMEE-JAIN}

\begin{abstract} Keywords: Kaolin suspension; Rheology; Friction factor.

\section{INTRODUÇÃO}

O escoamento eficiente de suspensão de caulim depende da otimização do projeto hidráulico, buscando sempre a menor soma dos custos fixos e variáveis. Neste caso, um dos parâmetros mais importantes é a perda de carga, a qual deve ser determinada com precisão, resultando no sistema de recalque mais econômico.
\end{abstract}

Estimating load losses due to friction in closed tubes is an important task in solving many practical problems in various branches of the engineering profession. Hydraulic design and the analysis of mineral suspension flow systems are two examples. Many authors consider the Churchill and Swamee-Jain models to be promising for evaluating losses due to friction in closed pipe conduits. To investigate the friction factor the Churchill and Swamee-Jain models were evaluated in terms of rheological data for the confined flow kaolin suspension galvanized iron pipe with 0.1 to 1.0 meter diameter at a flow range of 0.002 to $0.6 \mathrm{~m}^{3}$ per second. Both models showed a good representation of consistent friction factor with low relative percentage discrepancy providing estimated values for use in the design and analysis of kaolin flow systems.
O modelo Swamee-Jain (1976), citados por Porto [1], apresenta uma expressão geral dada pela Equação I que calcula o fator de atrito $(f)$ para faixa de rugosidade relativa de $1.10^{-6}$ a 0,001 e para numero de Reynold inferior a I.108 [2]. Onde Re é o numero de Reynolds e $\varepsilon / D$ é a rugosidade relativa.

'Departamento de Engenharia Química - DEQ, Universidade Federal do Pará - UFPA, Belém, PA, Brasil. E-mail: andremileo@ufpa.br ${ }^{2}$ Departamento de Engenharia Mecânica - DEM, Universidade Federal do Pará - UFPA, Belém, PA, Brasil.

${ }^{3}$ Programa de Pós-graduação em Engenharia de Recursos Naturais da Amazônia - PRODERNA, Universidade Federal do Pará - UFPA, Belém, PA, Brasil. 
O modelode Swamee-Jain é empregado para estimar o fator de atrito em regime turbulento apresentando boa convergência também para o regime laminar [3,4]. Tem a vantagem de se aplicar a qualquer regime de escoamento, 0 que simplifica a formulação dos problemas nos quais não se conhece a ordem de grandeza do número de Reynolds [5].

$$
f=\left\{\left(\frac{64}{R e}\right)^{8}+9,5\left[\operatorname{Ln}\left(\frac{1}{3,7} \cdot \frac{\varepsilon}{D}+\frac{5,74}{R e^{0,9}}\right)-\left(\frac{2500}{R e}\right)^{6}\right]^{-16}\right\}^{0,125}
$$

O modelo de Swamee-Jain é de aplicação geral e recentemente já vem sendo implementado pelo setor mineral como alternativa aos modelos clássicos. Com a validação do modelo de Swamee-Jain o setor mineral requer e incentiva sua aplicação e avaliação no processo de escoamento de diferentes suspensões minerais.

O modelo de Churchill [6] desenvolveu a expressão para o cálculo do fator de atrito nos três regimes de escoamento em tubos lisos e rugosos, através da combinação de equações existentes na literatura. Tem a vantagem de se aplicar a qualquer regime de escoamento, o que simplifica a formulação dos problemas nos quais não se conhece a ordem de grandeza do número de Reynolds [5]. A expressão do modelo de Churchill é dado pela Equação 2 em que os parâmetros A e B são expressos pelas Equações 3 e 4 respectivamente.

$$
\begin{aligned}
& f=8\left[\left(\frac{8}{R e}\right)^{12}+\frac{1}{(A+B)^{1,5}}\right]^{\frac{1}{12}} \\
& A=\left[2,457 \operatorname{Ln}\left(\frac{1}{\left(\frac{7}{R e}\right)^{0,9}+0,27 \frac{\varepsilon}{D}}\right)\right]^{16} \\
& B=\left(\frac{37530}{\operatorname{Re}}\right)^{16}
\end{aligned}
$$

A equação de Churchill foi tradicionalmente utilizada por Ellis e George [7], visando estimar o fator de atrito de fluido não-newtonianos em regime turbulento.

Os caulins são constituídos por argilo-minerais do grupo da caulinita, são eles a própria caulinita, a haloisita e metahaloisita, a nacrita e a diquita. Embora esses minerais tenham composição química similar, cada um deles possui importantes diferenças estruturais [8].

As suspensões aquosas de caulim são constituídas de partículas de ordem coloidal e com elevadas concentrações de sólidos. As propriedades reológicas de sistemas coloidais dependem da viscosidade do líquido dispersante, da concentração da fase dispersa, do tamanho e forma das partículas dispersas e das forças de interação, ou seja, do estado de estabilidade da suspensão [9].

O modelo de Herschel-Bulkley [ 10$]$ é empregado para relações não lineares observáveis na curva de escoamento onde $\mathrm{n}$ é o índice comportamento, na maioria dos casos práticos é inferior a $\mathrm{I}$.

O beneficiamento do minério de caulim é realizado através de via úmida sob a forma de suspensão, que necessita ser transportada, bombeada, ao longo do processo [II].

$\mathrm{Na}$ indústria papeleira, o caulim possui duas finalidades principais: carga e cobertura. No caso de ser utilizado como cobertura, a aplicação do caulim é efetuada sobre o papel na forma de polpas concentradas com até $65 \%$ em massa de sólidos [12].

Polpas com elevadas concentrações fornecem elevados valores de viscosidade. Daí a importância do fator de atrito para o correto dimensionamento do sistema de bombeamento de forma a evitar o superdimensionamento e consumo desnecessário de energia [13].

O trabalho tem como objetivo estimar o fator de atrito através do modelo de Churchill e de Swamee-Jain para o escoamento da suspensão de caulim com o intuito de contribuir para a melhoria na operação unitária de bombeamento no setor mineral além de avaliar a discrepância relativa percentual entre os modelos para o regime laminar e turbulento.

\section{MATERIAIS MÉTODOS}

\section{I Caracterização Química da Matéria-prima}

O caulim da suspensão aquosa, de origem da formação barreiras a margem do rio capim município de ipixuna do Pará produto do beneficiamento das indústrias da região, foi caracterizado através da técnica de fluorescência de raios-X utilizando espectrômetro WDS sequencial, modelo Axios Minerals PANalytical, com tubo de raios-X cerâmico e anodo de ródio.

\subsection{Característica da Suspensão de Caulim}

A suspensão de caulim a ser determinado o fator de atrito pelos modelos, apresenta um teor de sólidos de $65 \%$ em massa e uma massa especifica de $1,51 \mathrm{~g} / \mathrm{cm}^{3}$. $\mathrm{O} \mathrm{pH}$ da suspensão aquosa de caulim é de 5,3. Essas são condições operacionais em operação de bombeamento da indústria de papel em geral.

\subsection{Reologia}

Para obtenção de dados experimentais a suspensão de caulim foi ensaiada em viscosímetro VT 550 modelo Haake a $25^{\circ} \mathrm{C}$ para obtenção de curva de viscosidade aparente em função da taxa cisalhante de 0 a $700 \mathrm{~s}^{-1}$ com seus respectivos intervalos de erro. Após a obtenção experimental foi ajustado o modelo matemático de Hershel Bulkley o que 
possibilita a estimativa do fator de atrito pelos modelos de Churchill e de Swamee-Jain para as condições operacionais de escoamento.

\subsection{Condições Operacionais de Simulação de Escoamento}

Para utilização dos modelos de Churchill e de Swamee-Jain se adotou as especificações estruturais e operacionais para o sistema de bombeamento apresentado pela Tabela I.

Pela Tabela I o material da tubulação é ferro galvanizado e para a avaliação dos modelos se tem que para cada um dos cinco diâmetros hidráulicos da tubulação cilíndrica se avaliou escoamento do caulim numa faixa de vazão de 0,002 a $0,6 \mathrm{~m}^{3}$ por segundo. Essa rugosidade engloba $\circ$ ferro galvanizado [14].

\section{RESULTADOS E DISCUSSÃO}

\section{I Composição Química das Matérias-primas}

A Tabela 2 apresenta a composição química do caulim da suspensão ensaiada.

Pela Tabela 2 observa-se que o caulim, apresenta em sua composição $\mathrm{SiO}_{2}(46,64 \%)$. O resultado da fluorescência mostrou ainda um teor considerável de $\mathrm{Al}_{2} \mathrm{O}_{3}$ (37,75\%) característico do caulim utilizado como carga e cobertura na indústria de papel.

\subsection{Reologia}

A Figura I apresenta a curva experimental da viscosidade aparente versus taxa cisalhante para a suspensão de caulim com seus respectivos intervalos de erro.

Pela Figura I observa-se que a suspensão apresenta comportamento não newtoniano, pois a viscosidade decresce com o aumento da taxa cisalhante.

A Figura 2 apresenta o ajuste do modelo Herschel-Bulkley para os dados da Figura I.

De acordo com a Figura 2 observa-se o ajuste do modelo Herschel-Bulkley dado pela Equação 5 e representado pela curva sem intervalo de erro. Esse modelo apresenta

Tabela I. Condições Operacionais de escoamento

\begin{tabular}{lc}
\hline \multicolumn{1}{c}{ Tubulação: } & Ferro Galvanizado com rugosidade $\mathbf{0 , 1 5} \mathbf{~ m m}$ \\
\hline Faixa de Diâmetro: & 0,$1 ; 0,2 ; 0,3 ; 0,4 ; 0,5 ; 0,8$ e I,0 metros \\
Faixa de Vazão: & 0,002 a $0,6 \mathrm{~m}^{3}$ por segundo \\
\hline
\end{tabular}

Tabela 2. Análise Química do Caulim

\begin{tabular}{cccccccc}
\hline$\%$ & $\mathbf{S i O}_{2}$ & $\mathbf{A l}_{2} \mathbf{O}_{3}$ & $\mathbf{F e}_{2} \mathbf{O}_{3}$ & $\mathbf{N a}_{2} \mathbf{O}$ & $\mathbf{P}_{2} \mathbf{O}_{5}$ & $\mathbf{T i O}_{2}$ & P.F \\
\hline CAULIM & 46,64 & 37,75 & 0,53 & 0,23 & $0,1 \mathrm{I}$ & 0,39 & 14,33 \\
\hline
\end{tabular}

P.F - Perda ao Fogo. bom ajuste aos valores experimentais com um coeficiente de correlação de 0,979.

$\eta=\frac{0,4978}{\gamma}+0,057 \gamma^{0,8426-1}$

Pela Equação 5 é possível relacionar viscosidade com taxa cisalhante, sendo que essa função é útil para aplicação dos modelos de Churchill e de Swamee-Jain para estimativa do fator de atrito para as condições operacionais.

A viscosidade depende da taxa de cisalhamento pelo fato da suspensão de caulim ser um fluido não newtoniano 15, e a taxa de cisalhamento é função da vazão e do diâmetro da tubulação. A Figura 3 apresenta os resultados

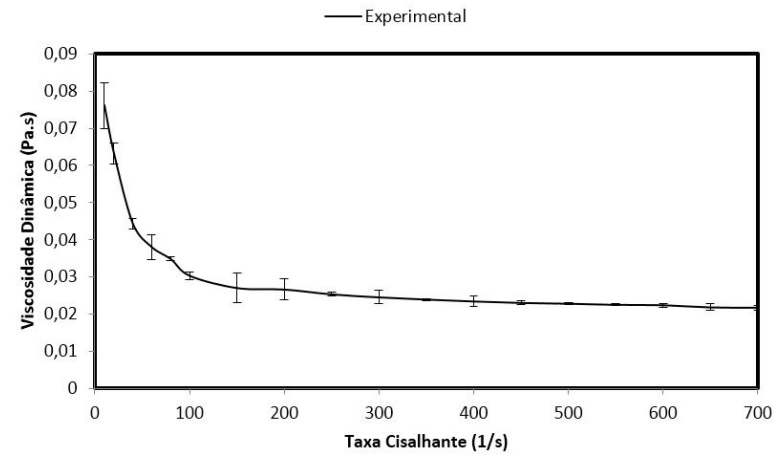

Figura I. Curva de viscosidade dinâmica versus taxa cisalhante.

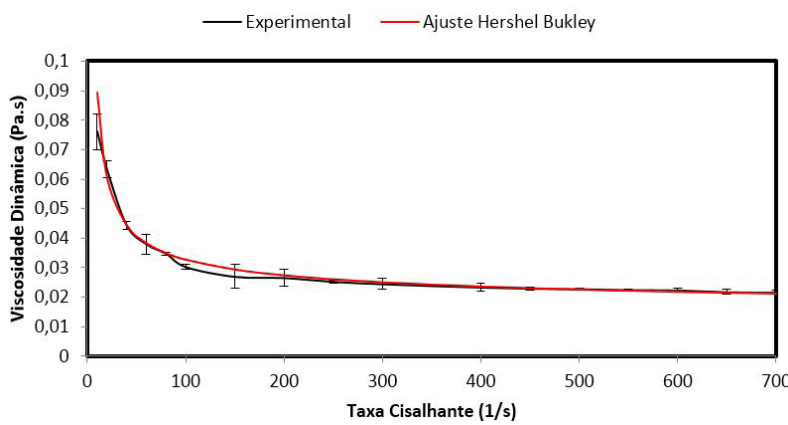

Figura 2. Curva de viscosidade dinâmica versus taxa cisalhante com ajuste de modelo Herschel-Bulkley.

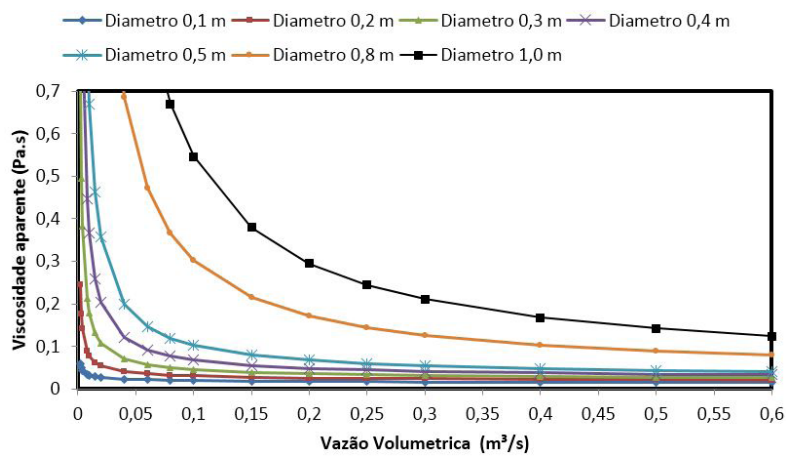

Figura 3. Curva de viscosidade versus vazão para diferentes diâmetros de tubulação. 
da viscosidade aparente $(\eta)$ em função da vazão volumétrica para a suspensão de caulim escoando na tubulação de ferro galvanizado com os respectivos diâmetros.

De acordo com a Figura 3 para todos os diâmetros se tem a diminuição da viscosidade aparente com o aumento da vazão devida o aumento da velocidade de escoamento.

Os valores de viscosidade acima de 0, I Pa.s são devido aos baixos valores de taxa cisalhante para as condições de escoamento nos maiores diâmetros de tubulação. $O$ modelo de Herschel-Bulkley ajustado é representativo também para essas baixas taxas cisalhantes.

Para as tubulações de ferro galvanizado de diâmetro hidráulico 0,$1 ; 0,2 ; 0,3 ; 0,4 ;$ e 0,5 metros a partir de $0,15 \mathrm{~m}^{3}$ por segundo se tem o estabelecimento do regime turbulento fazendo com que a viscosidade aparente se mantenha com baixo decréscimo numa faixa de 0,029 a 0,079 Pa.s.

Já para as tubulações de 0,8 e I,0 metros se tem - decréscimo acentuado da viscosidade aparente para o aumento de vazões até $0,25 \mathrm{~m}^{3}$ por segundo.

A Figura 4 apresenta os resultados do fator de atrito obtido pelo modelo de Churchill.

Pela Figura 4 para todos os diâmetros em analise o fator diminui com o aumento da vazão devido o aumento do numero de Reynolds.

Para as tubulações de maior diâmetro, para uma mesma vazão, observa-se uma menor velocidade de escoamento que por sua vez indica um menor Reynolds o que justifica o aumento do fator de atrito para os maiores diâmetros de tubulação. As oscilações nas curvas da Figura 4 para uma dada vazão são devido a transição do regime laminar para o turbulento.

A Figura 5 apresenta os resultados do fator de atrito obtido pelo modelo de Swamee-Jain.

A Figura 5 apresenta os resultados do fator de atrito estimado pelo modelo de Swamee-Jain. O fator de atrito diminui com o aumento da vazão de forma mais acentuada para os diâmetros de 0,8 e I,0 metros até uma vazão de $0,3 \mathrm{~m}^{3}$ por segundo, após essa vazão tende a estabilização numa faixa de fator de atrito de 0,045 a 0,03.

Para os diâmetros 0,1 a 0,5 metros a diminuição do fator de atrito é acentuada até a vazão de $0,2 \mathrm{~m}^{3}$ por segundo, após essa vazão tende a se estabilizar numa faixa de fator de atrito de 0,034 a 0,022.

A Figura 6 mostra o resultado da discrepância relativa percentual (DR\%) do modelo Swamee-Jain em relação ao modelo de Churchill na estimativa do fator de atrito para o escoamento da suspensão de caulim em regime laminar e turbulento.

Pela curva do regime laminar na Figura 6 o fator de atrito calculado pelo modelo de Churchill será numericamente igual ao calculado pelo modelo de Swamee-Jain sendo assim a discrepância relativa percentual é zero. Para o regime turbulentos ambos os modelos apresentaram pequena variação no valor do fator de atrito com discrepância relativa percentual inferior a $1 \%$.

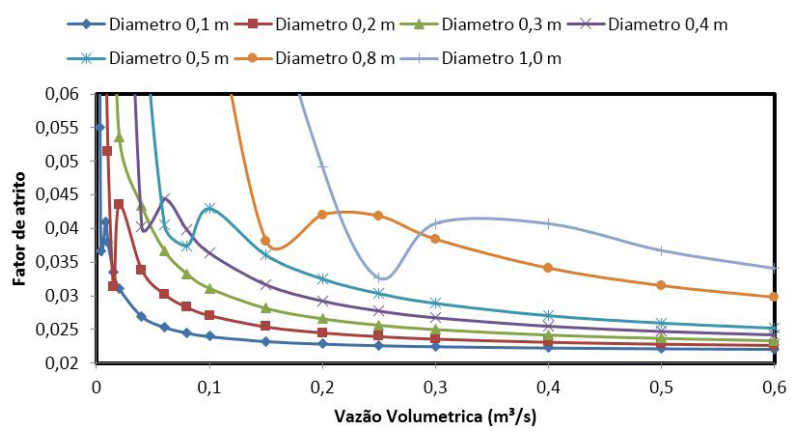

Figura 4. Fator de Atrito pelo modelo de Churchill.

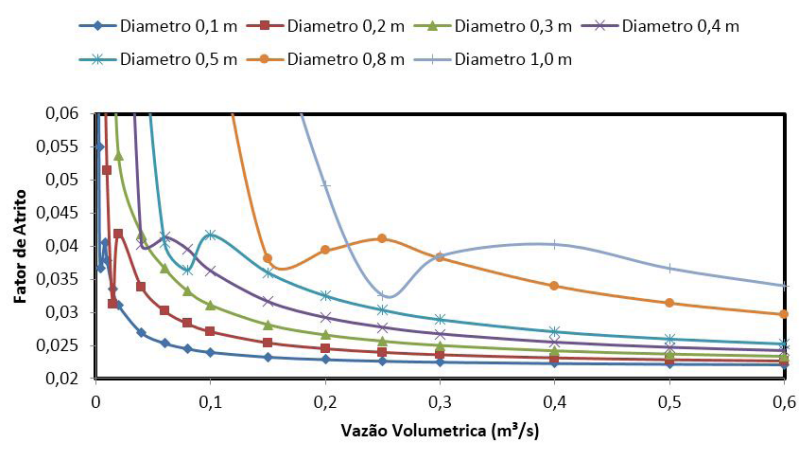

Figura 5. Fator de Atrito pelo modelo de Swamee-Jain.

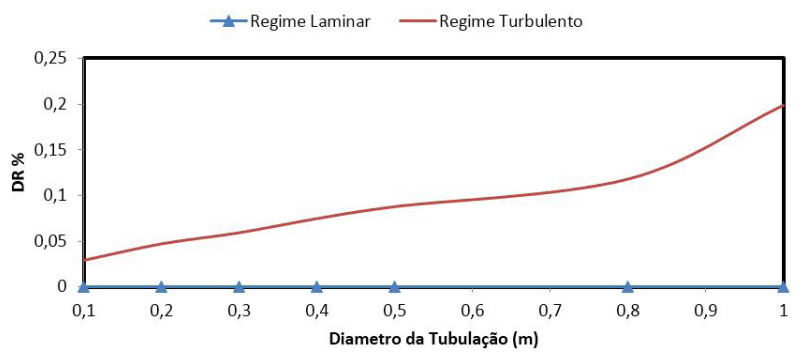

Figura 6. Discrepância relativa percentual do fator de atrito.

\section{CONCLUSÃO}

Os modelos de Churchill e de Swamee-Jain podem ser utilizados para a previsão do comportamento de fluxo da suspensão de caulim em regiões laminares e turbulentas de escoamento.

As tubulações de 0,8 e 1,0 metros de diâmetro possuem boa operação numa faixa de vazão de 0,2 a $0,6 \mathrm{~m}^{3}$ por segundo com numero de Reynolds respectivamente de 1302,07 a 17960,44 , pois nessa faixa se tem valores moderados tanto de fator de atrito quanto da velocidade de escoamento.

Para as tubulações de $0, \mathrm{I}$ a 0,5 metros de diâmetro a faixa de vazão de 0,05 a $0,2 \mathrm{~m}^{3}$ por segundo com numero de Reynolds respectivamente de II45,28 a 220705,7 apresentando boa operacionalidade devido aos valores moderados de fator de atrito e de velocidade de escoamento.

Os modelos de Churchill e de Swamee-Jain apresentaram os mesmos valores de fator de atrito para 
Silva et al.

todos os diâmetros de tubulação de ferro galvanizado no regime laminar.

Para o escoamento confinado da suspensão de caulim em regime turbulento nas tubulações de diferentes diâmetros, os valores de fator de atrito estimado pelos modelos de Churchill e de Swamee-Jain apresentaram baixa divergência com erro percentual inferior a $1 \%$.

A baixa diferença nos valores de fator de atrito obtidos pelos modelos de Churchill e de Swamee-Jain sustenta a possibilidade do uso não só do modelo de Churchill como também o de Swamee-Jain na simulação do fator de atrito para o escoamento confinado de caulim, possibilitando o projeto e o acompanhamento do escoamento de caulim de forma mais precisa contribuindo para a diminuição do consumo energético no setor mineral.

\section{Agradecimentos}

Os autores agradecem a CAPES pelo incentivo a pesquisa.

\section{REFERÊNCIAS}

I Porto RM. Hidráulica básica. 2. ed. São Carlos: EESC-USP; 1998.

2 Durand AIA, Segovia GIC, Bárcenas OF, Bravo VAGM. Evaluación de ecuaciones de factor de fricción explícito para tuberías. Educación en la Química. 2014;25:I28-134.

3 Winning HK, Coole T. Explicit friction factor accuracy and computational efficiency for turbulent flow in pipes. Flow, Turbulence and Combustion. 2013;90(I):I-27. http://dx.doi.org/10.1007/s 10494-0I2-9419-7.

4 Andrade L, Carvalho A. Análise da equação de Swamee-Jain para cálculo do fator de atrito. Revista Brasileira de Engenharia Agrícola e Ambiental. 200 I;5(3):554-557. http://dx.doi.org/I0.1590/S I4I5-4366200 I000300030.

5 Gomide R. Operações unitárias: fluidos na indústria. São Paulo; 1993. vol. 2.

6 Churchill SW. Friction factor equations spans all fluid flow regimes. Chemical Engineering. 1977;84:91-I02.

7 Ellis RC, George DS. Pratical interpretation on theology, annular displacing torces. How to avoid by passing mud during primary cementing. World Oil. 1977:64-9.

$8 \mathrm{Hu}$ Y, Liu X. Chemical composition and surface properties of kaolins. Minerals Engineering. 2003; I6(I I):I279- I 284. http://dx.doi.org//0.1016/j.mineng.2003.07.006.

9 Luz AB, Chaves AP. Processo de beneficiamento de caulim com estabilização dos íons ferrosos. Patente PI 9803302. 2000.

10 Herschel WH, Bulkley T. Measurement of consistency as applied to rubber-benzene solutions. Proceedings of the American Society for Testing and Materials. 1926;26(2):62I-633.

I I Varela JJ, Gliese R, Petter CO, Peixoto CA. Controle de qualidade no processamento de polpas de caulim utilizando propriedades óticas. Revista Escola de Minas. 2005;58(3):20I-206. http://dx.doi.org/I0.1590/S037044672005000300004.

12. Pettendorfer EPO. Efeito de dispersantes na reologia de polpas de caulim para a indústria de papel [dissertação de mestrado]. Rio de Janeiro: Departamento de Ciência dos Materiais e Metalurgia, Pontifícia Universidade Católica do Rio de Janeiro; 1999.

13 Vlasak P, Chara Z. Laminar and turbulent flow experiments with yield power-law slurries. Powder Technology. 1999; 104(3):200-206. http://dx.doi.org/10.1016/S0032-5910(99)00095-9.

14 Azevedo JM No, Fernandez Y, Fernandez M, Araujo R, Ito AE. Manual de hidráulica. 8. ed. São Paulo: Blücher; 1998. $669 \mathrm{p}$.

Recebido em: 2 Jun. 2016

Aceito em: 10 Ago. 2016 